1

2

3

\title{
Effect of High Glucose on Human Alveolar Macrophage Phenotype and Phagocytosis of Mycobacteria
}

Jesse Vance ${ }^{1}$, Andres Santos ${ }^{1}$, Laura Sadofsky², Alyn Morice ${ }^{2}$, and Jorge Cervantes ${ }^{1 *}$

${ }^{1}$ Paul L. Foster School of Medicine, Texas Tech University Health Sciences Center, El Paso, TX, U.S.A.; ${ }^{2}$ The Hull York

Medical School, University of Hull, Hull, U.K.

*Corresponding author: Jorge Cervantes

Paul L. Foster School of Medicine, Texas Tech University Health Sciences Center, El Paso.

5001 El Paso Dr., El Paso, TX 79905. Tel: 915-215-4672 Fax: 915-783-1715

e-mail: jorge.cervantes@ttuhsc.edu

\begin{abstract}
Diabetes mellitus (DBM) reduces immunological activity and increases susceptibility to various infections, including tuberculosis. Human alveolar macrophage (hAM) functions are altered in DBM.

To mimic hyperglycemic conditions in the lung alveolus, we co-cultured a hAM cell line (Daisy cell line) with human umbilical vein endothelial cells (HUVEC) for 48 hours in the presence of culture media alone, normal glucose $(5 \mathrm{mM})$, and high glucose (22 mM). Using flow cytometry, immunophenotype characterization included cell surface markers CD 11c, CD14, CD16, CD86, CD163, CD169, CD206, CX3CR-1, CSF-1R, and MMP9. Phagocytic function was measure by immunofluorescence microscopy at 24 hours after inoculation of cells with GFP-expressing Mycobacterium smegmatis.

Direct exposure of AMs to high glucose and exposure in the co-culture system yields different results for the same phenotypic markers. Matrix metalloproteinase-9 (MMP9) expression was increased under both conditions. CD169 and CX3CR1 expression were decreased when AMs were exposed directly to high glucose but increased under co-culture. Immunofluorescence assay revealed that phagocytosis decreased in AMs directly exposed to an increase of glucose from 2.5 $\mathrm{mM}$ to normal glucose $(5 \mathrm{mM})$, yet AMs under co-culture did not show decreased phagocytosis until concentrations were raised to $25 \mathrm{mM}$.
\end{abstract}

Alteration in the expression of certain receptors may contribute to defective sentinel function of AMs, promoting s susceptibility to tuberculosis in a diabetic host. Variability in cell surface marker expression under direct glucose exposure compared to exposure via co-culture reveals that cell signaling between endothelial cells and AMs may play a crucial role in the phenotypic expression of AMs.

Keywords: Diabetes; alveolar macrophages; tuberculosis; mycobacteria 


\section{INTRODUCTION}

Tuberculosis (TB) is one of the oldest known communicable diseases and, as it has throughout history, it continues to persist as a major global health problem [1,2]. According to the World Health Organization (WHO), pre-existing diabetes mellitus increases the risk of developing active TB three-fold [2]. While historically type II diabetes was considered a disease of wealthy countries with food excess, the trends show that over the last 15 years the incidence of diabetes mellitus (DBM) has been rising steadily across the globe, affecting high- and low-income countries alike [3,2]. With these conditions occurring together at an increasing rate, it is pivotal to improve our understanding of the complex interplay that makes individuals more susceptible to such a deadly disease.

DBM is a chronic disease that occurs either when the pancreas does not produce enough insulin or when the body cannot effectively use the insulin it produces. Even though the pancreas attempts to compensate for decreased effectiveness of insulin, hyperglycemia, or raised blood sugar, still occurs. This state of increased blood glucose (sugar) leads to serious damage to many of the body's systems over time. Particularly, DBM reduces immunological activity and increases susceptibility to various infections, including TB [4]. Population data clearly demonstrates this increased risk and in vitro studies have shown that elevated glucose concentrations directly affect human alveolar macrophage (hAM) functions, such as pathogen phagocytosis, removal of senescent cells, and tissue repair $[2,5,6]$. These alveolar macrophages are responsible for the clearance of invading microbes in the lungs and their dysfunction leads to an increased risk of pulmonary infection, including TB [7,8]. The phagocytic function of hAMs and the expression of adhesion molecules has been studied elsewhere but the overall mechanism of impairment has not yet been fully elucidated.

We herein aimed to investigate the effects of increasing glucose concentrations on the phenotype and function of the hAM. A unique co-culture system was designed to simulate the interstitial environment in which the hAM resides. hAMs were indirectly exposed to increasing glucose concentrations via a layer of endothelial cells, similar to what occurs in DBM. [8]. Finally, hAMs that had been exposed to high levels of glucose for an extended period were isolated and inoculated with Mycobacterium smegmatis to assess changes in phagocytic function. 


\section{MATERIAL AND METHODS}

\section{Co-culture system and cell assay conditions}

The overall approach to this experiment was to create a variety of conditions in which hAMs can be exposed to glucose. Direct exposure of hAMs to increasing glucose concentrations was then compared to in-direct exposure via a co-culture system. A visual representation of the co-culture system is depicted in Figure 1. In this system, hAMs are separated by the contents of the upper well by a layer of endothelial cells and a micropore membrane. This system tries to replicate the internal environment of the alveolus, as AMs are not directly exposed to elevated blood glucose concentrations.

The hAM cell line (Daisy cell line), was maintained in RPMI-1640 medium supplemented with 10\% Fetal Bovine Serum, and $1 \%$ non-essential amino acids and incubated at $37^{\circ} \mathrm{C}$ under a $5 \% \mathrm{CO}_{2}$ atmosphere. Human umbilical vein endothelial cells (HUVEC) were maintained in Endothelial Cell Growth Medium (Cell Applications, Inc.) and incubated at $37^{\circ} \mathrm{C}$ under $5 \% \mathrm{CO}_{2}$ atmosphere. D-glucose solution (Sigma) was added to wells to create various glucose conditions. Concentrations tested include standard media $(2.5 \mathrm{mM})$, normal glucose $(5 \mathrm{mM})$, and high glucose $(22 \mathrm{mM})$. HUVEC cells, in co-culture with hAMs using a collagen-coated transmembrane wells (Cornins), or hAMs alone were exposed to glucose concentrations described above. Cells were incubated for 48 hours under $5 \% \mathrm{CO}_{2}$ at $37^{\circ} \mathrm{C}$, before being washed and scrapped for flow cytometry.

\section{Flow cytometry}

Flow cytometry was performed using a BD AccuriM C6 Flow Cytometer and results were analyzed using Flow-Jo V10 software. Immunophenotype characterization was performed to analyze expression cell surface markers CD11c, CD14, CD16, CD86, CD163, CD169, CD206, Cx3CR-1, CSF-1R, and MMP9. Histograms were analyzed for changes in cell surface marker expression across varying glucose levels for each marker.

\section{Phagocytic assay}

A separate plate with a glass slip cover placed at the bottom of each well was used for phagocytic assays. Upon incubation under different glucose concentrations, hAMs were inoculated with GFP-expressing M. smegmatis, and then incubated for an additional 24 hours. Cells were fixed, permeabilized (Intracellular Staining Kit, Novus), and lysosomes stained with a LAMP1 antibody (DSHB, IA). The nuclei were stained using DAPI. Cells were examined under fluorescence microscopy, and images were captured with Metamorph 7.8 (Molecular Devices Inc.) and processed using Image J (1.50i, NIH, U.S.A). GFP-expressing 
mycobacteria that co-localized to the phagolysosome were identified by a yellow signal on the composite images. The number of cells containing mycobacteria co-localized to the phagolysosome were counted from a random sample of at least 40 AMs.

\section{Statistical analysis}

Phagocytosis data across the different conditions was compared using a paired $t$ test after testing for normality (Shapiro-Wilk normality test). GraphPad Prism 7.01 was used for statistical calculations.

\section{RESULTS}

Direct exposure of AMs to high glucose and exposure via co-culture with endothelial cells yield different phenotypic changes in AMs

First, we verified that Daisy cells were phenotypically hAM cells using flow cytometry. Previous studies have shown that hAMs are M2 macrophages demonstrating presence of cell surface markers CD163 and CD206 [9-11] After confirming that Daisy cells phenotype function as an adequate marker for hAMs, other cell surface markers were investigated [12].

Table 1 summarizes the changes in phenotypic expression of all cell surface markers at high glucose (22 $\mathrm{mM})$ compared to media. The table allows for comparison between groups (hAMs alone vs. co-culture system). Interestingly, direct exposure of hAMs to high glucose and exposure via the co-culture system yields different results for the same markers. For instance, CX3CR1 expression decreases slightly under high glucose conditions when directly exposed and increases greatly when exposed indirectly via the co-culture system. Expression of CD169 decreases under both conditions, but the decrease is greater under direct glucose exposure conditions. Expression of MMP9 increases greatly when directly exposed and only increases slightly in the co-culture system. See Supplemental figure 1 (Suppl 1.) for histogram comparison.

\section{Phagocytosis of mycobacteria by AMs is impaired only at HG concentrations when exposure occurs in co-culture with} endothelial cells

We then compared the effect of the different glucose concentrations on hAMs when cells where exposed directly or via coculture with HUVEC cells. hAMs directly exposed to both normal glucose conditions (5 mM) and high glucose conditions (22 $\mathrm{mM}$ ) show a decrease in phagocytosis. ( $\mathrm{p}=0.01)$ (Figure 2). Similarly, hAMs in co-culture had decreased phagocytosis at high glucose concentrations $(22 \mathrm{mM})$ compared to media $(\mathrm{p}=0.01)$. Interestingly, hAMs appear to have decreased phagocytic 


\section{DISCUSSION}

It is well documented that DBM increases the risk of developing active tuberculosis, with up to $30 \%$ of TB patients presenting with concomitant DBM type 2 [2,13]. With approximately $1 / 3$ of the world's population infected with latent TB and the incidence of DBM steadily on the rise, a better understanding of the immunologic mechanisms by which DM predisposes some to developing active TB is needed [3,2]. Our experiments aimed to evaluate the response of hAMs to glucose directly and in a co-culture system with endothelial cells. This unique co-culture system allows for indirect exposure of hAMs to glucose, just as they would be in vivo [6]. Previous reports focusing on hAMs and rat AMs responses to direct exposure of elevated glucose concentrations includes studies using glucose concentrations as high as $33 \mathrm{mM}$ [14,5]. This concentration of $33 \mathrm{mM}$ blood glucose is equivalent to a HbA1c of roughly $22 \%$ while a diagnosis of DBM can be made at a level $\geq 6.5 \%$ [15]. This methodology represents an extreme environmental insult to the AM and may not be indicative of what happens in vivo. To make matters even more complicated, it is estimated that the alveolar environment will have an even further decreased amount of glucose due to a combination of mechanisms that maintain the alveolus at a concentration as much as 10 -fold lower than plasma [16]. For this reason, our approach may be able to better approximate such conditions.

Phenotypically, hAMs are characterized by the expression of CD206 (macrophage mannose receptor) and CD163 (macrophage scavenger receptor) [9]. This natural state of hAMs indicates M2 polarization, the alternative pathway that suppresses bacterial killing and promotes remodeling, potentiates fibrosis, and ultimately damages surrounding tissue if left unchecked [7]. A careful examination of various cell surface markers known to be expressed on M1 and M2 macrophages can help determine how hAMs react to increasing glucose concentrations and give insight into why patients with diabetes may be unable to mount a bacterial killing response when confronted with mycobacteria. It has been shown that M2 activated macrophages promote intracellular bacteria survival, which is how M. tuberculosis remains latent in the lungs [17].

Lung interstitial macrophages are involved in the recruitment of immune cells after infection with M. tuberculosis [18]. These cells typically express CX3CR1, a cell surface chemokine receptor responsible for migration and adhesion [19]. In co-culture, when indirectly exposed to high glucose levels, hAMs greatly increased their expression of CX3CR1 (Suppl 1.A.). This increase in expression may signal hAMs to move to a site of infection or inflammation and begin phagocytosis or tissue repair. 
Interestingly, when directly exposed to high glucose, hAMs downregulate this chemokine receptor. This downregulation could potentially lead to decrease migration to the site of infection. Because the two experimental conditions result in different phenotypic expression of CX3CR1, it should be considered that cell signaling between HUVEC cells and hAM cells may have played role. Further investigation of supernatants may give some insight into the mechanism behind this change.

Expression of CD169, a pathogen recognition receptor, is seen in murine and hAMs [20,21]. This marker is increased during the inflammatory process and indicates an M2 polarization of murine and hAMs [9,22]. Expression of CD169 on hAMs under direct glucose exposure drastically decreased whereas exposure via the co-culture system lead to an increase in expression, suggesting again an M2 response (Suppl. 1B).

MMP9 is an endopeptidase involved in the cleavage of certain cell surface receptors and release of cytokines. It is involved in numerous cellular functions including apoptosis. Expression of high levels of MMP9 has been indicated in poor outcomes during TB infection and has been isolated in hAMs from the lungs of TB patients [23,24]. Upregulation of MMP9 in response to elevated glucose concentrations demonstrates a shift to M2 macrophage polarization [25]. This alternative activation of macrophages favors bacterial survival [9]. Prolonged activation will also lead to lung remodeling and damage, further complicating active TB infection[7]. Expression of MMP9 increases greatly when directly exposed and only increases slightly in the co-culture system (Suppl. 1 C).

Phagocytic function of hAMs was decreased when directly exposed to normal glucose but only decreases in the co-culture system under high glucose conditions. HUVEC cells may create a barrier to some glucose reaching hAMs, but it is more likely that the difference in phagocytic function is due to cell-to-cell signaling between HUVEC and hAMs. Cells of the co-culture system were more likely to localize internalized M. smegmatis to the lysosome where bacterial killing could take place. This again highlights the drawbacks of other research that examines phagocytosis of AMs directly exposed to glucose [5], as direct exposure even to normal glucose concentrations may cause a decrease in phagocytosis.

In conclusion, exposing hAMs directly to glucose and indirectly via a co-culture system yields different phenotypic and functional changes. High glucose concentrations increased expression of certain M2 polarization markers such as MMP9 and CD169 in hAMs in co-culture, potentially creating favorable conditions for the survival of mycobacteria. Expression of chemokine receptor CX3CR1 responds differently depending on whether they were exposed directly or via the co-culture system. Phagocytic assay further demonstrates a decreased ability of hAMs under high glucose to clear internalized $M$. smegmatis. Variability in cell surface marker expression and phagocytic function of macrophages under direct glucose exposure 
compared to exposure via co-culture reveals that cell signaling between endothelial cells and hAMs may play a crucial role in the function of hAMs.

\section{Acknowledgements}

Special thanks to Dr. Nicolai van Oers (UT Southwestern) for supplying the GFP-expressing M. smegmatis.

\section{Conflict of interest}

None

\section{References}

1. Donoghue HD, Spigelman M, Greenblatt CL, Lev-Maor G, Bar-Gal GK, Matheson C, Vernon K, Nerlich AG, Zink AR (2004) Tuberculosis: from prehistory to Robert Koch, as revealed by ancient DNA. Lancet Infect Dis 4 (9):584592. doi:10.1016/S1473-3099(04)01133-8

2. Global tuberculosis report 2018 (2018).

3. McCarthy M (2014) Nearly one in 10 US residents has diabetes, CDC reports. BMJ 348:g3962. doi:10.1136/bmj.g3962

4. Hall JE (2016) Guyton and Hall textbook of medical physiology. 13th edition. edn., 5. Montoya-Rosales A, Castro-Garcia P, Torres-Juarez F, Enciso-Moreno JA, Rivas-Santiago B (2016) Glucose levels affect LL-37 expression in monocyte-derived macrophages altering the Mycobacterium tuberculosis intracellular growth control. Microb Pathog 97:148-153. doi:10.1016/j.micpath.2016.06.002

6. Brennan AL, Gyi KM, Wood DM, Johnson J, Holliman R, Baines DL, Philips BJ, Geddes DM, Hodson ME, Baker EH (2007) Airway glucose concentrations and effect on growth of respiratory pathogens in cystic fibrosis. J Cyst Fibros 6 (2):101-109. doi:10.1016/j.jcf.2006.03.009

7. Kumar V, Abbas AK, Aster JC (2015) Robbins \& Cotran Pathologic Basis of Disease. Robbins Pathology, 9th edn. Elsevier Health Sciences, London

8. Young B, Woodford P, O'Dowd G (2014) Wheater's Functional Histology A Text and Colour Atlas

9. Chavez-Galan L, Olleros ML, Vesin D, Garcia I (2015) Much More than M1 and M2 Macrophages, There are also CD169(+) and TCR(+) Macrophages. Front Immunol 6:263. doi:10.3389/fimmu.2015.00263

10. Cai Y, Sugimoto C, Arainga M, Alvarez X, Didier ES, Kuroda MJ (2014) In vivo characterization of alveolar and interstitial lung macrophages in rhesus macaques: implications for understanding lung disease in humans. J Immunol 192 (6):2821-2829. doi:10.4049/jimmunol.1302269

11. Roszer T (2015) Understanding the Mysterious M2 Macrophage through Activation Markers and Effector Mechanisms. Mediators Inflamm 2015:816460. doi:10.1155/2015/816460

12. Hayman Y, Hart S, Morice A (2014) Characterisation of a new Daisy cell line representative of human alveolar macrophage (hAM). European Respiratory Journal 38

13. Ruslami R, Aarnoutse RE, Alisjahbana B, van der Ven AJ, van Crevel R (2010) Implications of the global increase of diabetes for tuberculosis control and patient care. Tropical medicine \& international health : TM \& IH 15 (11):1289-1299. doi:10.1111/j.1365-3156.2010.02625.x

14. Li H, Peng W, Jian W, Li Y, Li Q, Li W, Xu Y (2012) ROCK inhibitor fasudil attenuated high glucose-induced MCP-1 and VCAM-1 expression and monocyte-endothelial cell adhesion. Cardiovasc Diabetol 11:65.

doi:10.1186/1475-2840-11-65 
15. American Diabetes A (2016) Standards of Medical Care in Diabetes-2016 Abridged for Primary Care Providers. Clin Diabetes 34 (1):3-21. doi:10.2337/diaclin.34.1.3

16. de Prost N, Saumon G (2007) Glucose transport in the lung and its role in liquid movement. Respir Physiol Neurobiol 159 (3):331-337. doi:10.1016/j.resp.2007.02.014

17. Italiani P, Boraschi D (2014) From Monocytes to M1/M2 Macrophages: Phenotypical vs. Functional Differentiation. Front Immunol 5:514. doi:10.3389/fimmu.2014.00514

18. Hall JD, Kurtz SL, Rigel NW, Gunn BM, Taft-Benz S, Morrison JP, Fong AM, Patel DD, Braunstein M, Kawula TH (2009) The impact of chemokine receptor CX3CR1 deficiency during respiratory infections with Mycobacterium tuberculosis or Francisella tularensis. Clin Exp Immunol 156 (2):278-284. doi:10.1111/j.1365-2249.2009.03882.x 19. Wolf Y, Yona S, Kim KW, Jung S (2013) Microglia, seen from the CX3CR1 angle. Front Cell Neurosci 7:26. doi:10.3389/fncel.2013.00026

20. Yu YR, Hotten DF, Malakhau Y, Volker E, Ghio AJ, Noble PW, Kraft M, Hollingsworth JW, Gunn MD, Tighe RM (2016) Flow Cytometric Analysis of Myeloid Cells in Human Blood, Bronchoalveolar Lavage, and Lung Tissues. Am J Respir Cell Mol Biol 54 (1):13-24. doi:10.1165/rcmb.2015-01460C

21. Cicek BB, Khanna KM (2016) Discovery of a novel subset of lung resident macrophages that regulate pathogen clearance and immune homeostasis after respiratory infection. The Journal of Immunology 196:68.6168.61

22. Bogie JF, Boelen E, Louagie E, Delputte P, Elewaut D, van Horssen J, Hendriks JJ, Hellings N (2017) CD169 is a marker for highly pathogenic phagocytes in multiple sclerosis. Mult Scler:1352458517698759. doi:10.1177/1352458517698759

23. Wang XW, Liu JJ, Wu QN, Wu SF, Hao DJ (2017) The in vitro and in vivo effects of microRNA-133a on intervertebral disc destruction by targeting MMP9 in spinal tuberculosis. Life Sci 188:198-205. doi:10.1016/j.Ifs.2017.07.022

24. Coussens A, Timms PM, Boucher BJ, Venton TR, Ashcroft AT, Skolimowska KH, Newton SM, Wilkinson KA, Davidson RN, Griffiths CJ, Wilkinson RJ, Martineau AR (2009) 1alpha,25-dihydroxyvitamin D3 inhibits matrix metalloproteinases induced by Mycobacterium tuberculosis infection. Immunology 127 (4):539-548. doi:10.1111/j.1365-2567.2008.03024.x 25. Jager NA, Wallis de Vries BM, Hillebrands JL, Harlaar NJ, Tio RA, Slart RH, van Dam GM, Boersma HH, Zeebregts CJ, Westra J (2016) Distribution of Matrix Metalloproteinases in Human Atherosclerotic Carotid Plaques and Their Production by Smooth Muscle Cells and Macrophage Subsets. Mol Imaging Biol 18 (2):283291. doi:10.1007/s11307-015-0882-0 


\title{
FIGURE LEGENDS
}

\section{Figure 1. Co-culture System resembling the Pulmonary Alveolus.}

\begin{abstract}
A unique co-culture system was designed seeking to replicate the environment and the interstitial conditions occurring in DBM.
Endothelial cells are directly exposed to high blood glucose, while hAMs in the alveolar space interact with the endothelium indirectly. Exposure to HG via co-culture, allows cell signaling between endothelial cells and hAMs.
\end{abstract}

\section{Figure 2. Percentage of Phagocytosis of M. smegmatis in hAMs}

Phagocytic function of hAMs was evaluated by immunofluorescence microscopy after inoculating hAMs upon incubation with the different glucose concentrations directly or via co-culture system, with a GFP-expressing Mycobacterium smegmatis, and then incubated for an additional 24 hours.

*p-value $=0.01$ 
Table 1. Phenotypic changes in hAM cells upon exposure to high glucose (22 mM)

\begin{tabular}{lcc}
\hline Cell Marker & Co-culture & hAM Alone \\
\hline CD16 & NC & NC \\
CD86 & NC & NC \\
MMP9 & $\uparrow$ & $\uparrow \uparrow$ \\
CD14 & NC & NC \\
CD206 & NC & NC \\
CD169 & $\uparrow$ & $\downarrow \downarrow$ \\
Cx3cr1 & $\uparrow \uparrow$ & $\downarrow$ \\
CD163 & NC & NC \\
CD11C & NC & NC \\
CDF-1R & NC & NC \\
\hline
\end{tabular}

NC: No change; $\uparrow:$ increase; $\downarrow$ : decrease 
Suppl 1. Expression of (A) CX3CR1 (B) CD169, and (C) MMP9 on hAMs in Media and High Glucose in co-culture vs. direct exposure. 

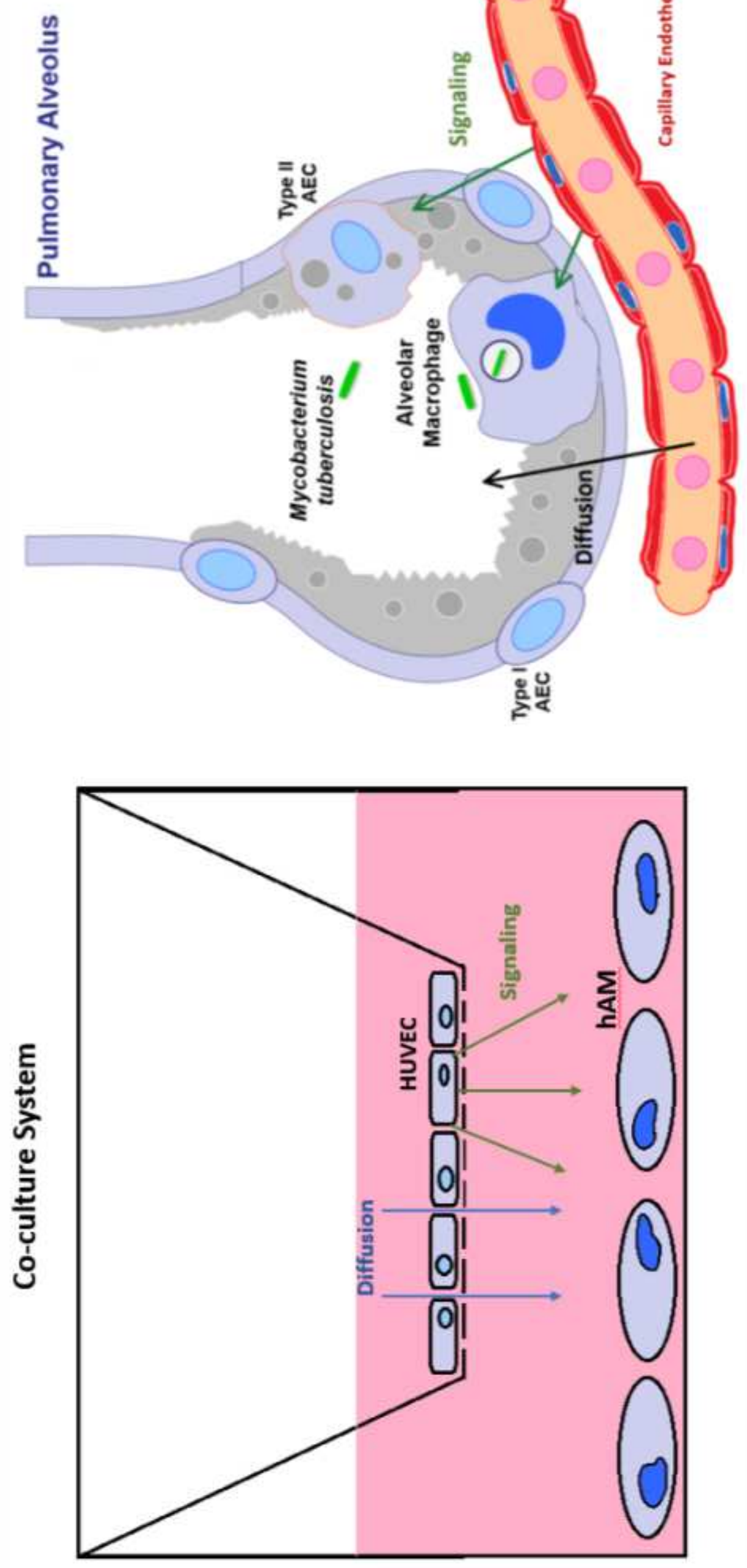


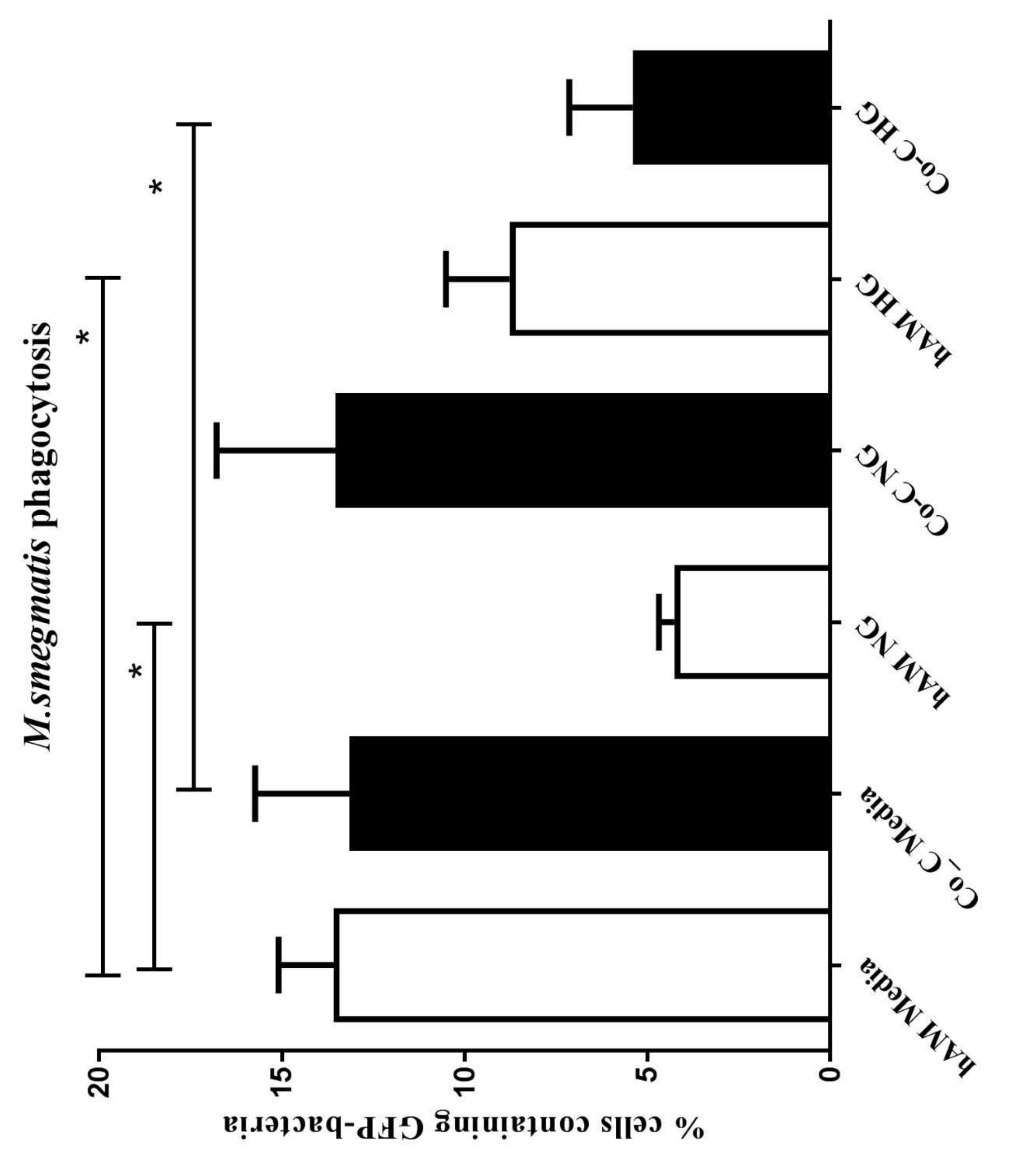

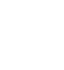

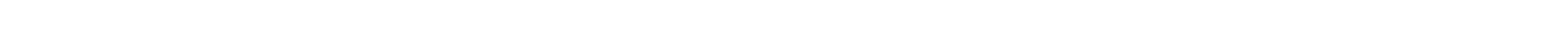


Click here to access/download Supplementary Material - To be Published Supplemental 1.png 